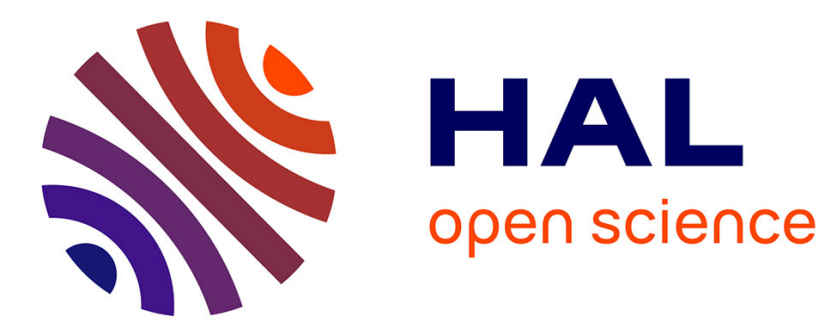

\title{
La phagothérapie expérimentale à l'aube du xxie siècle
}

Laurent Debarbieux

\section{To cite this version:}

Laurent Debarbieux. La phagothérapie expérimentale à l'aube du xxie siècle. Médecine et Maladies Infectieuses, 2008, 38 (8), pp.421 - 425. 10.1016/j.medmal.2008.06.014 . pasteur-03165488

\section{HAL Id: pasteur-03165488 \\ https://hal-pasteur.archives-ouvertes.fr/pasteur-03165488}

Submitted on 10 Mar 2021

HAL is a multi-disciplinary open access archive for the deposit and dissemination of scientific research documents, whether they are published or not. The documents may come from teaching and research institutions in France or abroad, or from public or private research centers.
L'archive ouverte pluridisciplinaire HAL, est destinée au dépôt et à la diffusion de documents scientifiques de niveau recherche, publiés ou non, émanant des établissements d'enseignement et de recherche français ou étrangers, des laboratoires publics ou privés.

\section{(이)(\$)}

Distributed under a Creative Commons Attribution - NonCommercial - NoDerivatives| 4.0 
La phagothérapie expérimentale à l'aube du XXI ${ }^{\text {ème }}$ siècle Experimental phage therapy in the beginning of the $\mathrm{XXI}^{\text {rst }}$ century

\author{
L Debarbieux \\ Département de Microbiologie \\ Unité de Biologie Moléculaire du Gène chez les Extrêmophiles \\ Institut Pasteur \\ 75724 Paris Cedex 15 \\ deblaure@pasteur.fr
}

Titre courant : la phagothérapie au XXI ${ }^{\text {ème }}$ siècle 
Résumé :

Après le déclin de l'utilisation de la phagothérapie en Occident au milieu du XX ${ }^{\text {ème }}$ siècle, la communauté scientifique occidentale s'est désintéressée de cette approche thérapeutique. Puis, à la fin du $\mathrm{XX}^{\mathrm{eme}}$ siècle, poussés par la nécessité de trouver des solutions originales à l'émergence rapide de la résistance aux antibiotiques, certains scientifiques se sont à nouveau tournés vers la phagothérapie. Si entre les années 1980 et 2000, le nombre des articles scientifiques est resté faible, on a pu noter une augmentation notable de ceux-ci au cours des 5 dernières années. Cette revue a pour but de synthétiser les articles les plus récents afin d'en faire ressortir les principaux acquis et les questions encore en suspens.

Mots-clés : bactériophages, thérapie, antibiotiques, résistance

Summary :

After that phage therapy was abandonned in Occident in the middle of the $\mathrm{XX}^{\text {th }}$ century, this scientific community disregarded this therapeutic approach. Then, at the end of the $\mathrm{XX}^{\text {th }}$ century, pushed by the neccesity to find new solutions to the rapid rising of antibiotic resistant bacteria, some scientists took a new look at phage therapy. If between 1980 and 2000, the number of scientific articles was low, it was noted a subtantial increase during the past 5 years. This review synthesizes the most recent papers to point out new data and questions still to be addressed.

Keywords: phages, therapy, antibiotics, resistance

\section{Les bactériophages : définitions et rappels}

Les bactériophages font partie de la grande famille des virus. Ils n'ont de particulier que leur spécificité, à savoir qu'ils infectent exclusivement des bactéries. En tant que virus, ils ont un mode de propagation classique : ils se multiplient au détriment des bactéries. On distingue deux classes de phages : les phages lytiques et les phages tempérés. Ces derniers sont très connus grâce au développement des premiers outils génétiques issu de l'utilisation du phage $\lambda$. Cependant il ne faut pas perdre de vue que l'ensemble des phages tempérés ne représentent qu'une faible partie (10 à 20\%) des phages présents dans la nature. Ce sont les chercheurs qui dans leur quête d'outils génétiques ont favorisé l'isolement de tels phages. Ainsi donc, la très grande majorité des phages sont dits lytiques. Leur représentant le plus connu est sans doute le phage T4, infectant Escherichia coli, tout comme le phage $\lambda$.

La différence entre un phage tempéré et un phage lytique vient du fait que le phage tempéré possède la capacité d'intégrer son génome au sein du génome de son hôte et de rester ainsi «silencieux », jusqu'au jour où sous l'influence de certains signaux le génome du phage s'excise, et entame alors un cycle lytique complet identique au cycle des phages strictement lytiques. C'est lors de l'étape de l'excision que le phage tempéré peut «emporter » avec lui une partie du génome de la bactérie-hôte. Comme l'ADN véhiculé est pris plus ou moins au hasard dans le génome bactérien, et que les phages tempérés peuvent infecter des hôtes pylogénétiquement proches, il s'en suit alors des remaniements génétiques. Ainsi un phage tempéré peut servir de véhicule à des fragments d'ADN bactériens pour le plus grand bonheur des généticiens. Aujourd'hui avec le séquençage de nombreux génomes bactériens on peut mesurer l'impact réel que les bactériophages tempérés ont sur la dynamique des génomes. Pour en terminer avec les phages tempérés, il est nécessaire de préciser que lorsqu'ils infectent un hôte, ces phages entrent dans un cycle de recombinaison de leur génome (cycle lysogénique), ou démarrent immédiatement un cycle lytique. En réalité les observations effectuées en laboratoire montrent que seule une petite fraction des phages tempérés 
« choisi »d'intégrer son génome. La très grande majorité se comporte comme des phages strictement lytique. Pour simplifier et conclure sur la nature des phages, on peut retenir que tous les phages sont lytiques et que seuls quelques-uns manifestent un caractère tempéré.

Le processus infectieux d'un bactériophage débute par la rencontre entre ce dernier et une bactérie hôte. La probabilité de cette rencontre est facteur du nombre de chacun des partenaires. On sait, par des expériences en laboratoire que, lorsque le rapport entre le nombre de bactéries et le nombre de bactériophages n'est pas optimal, l'infection peut ne pas avoir lieu. Une fois que l'infection a débuté, le nombre de phages croît chaque fois qu'une cellule est lysée et, au cours du temps, le nombre de bactéries hôtes diminue, jusqu'à devenir très faible, voire trop faible. Durant cette étape, les bactéries sont soumises à une pression de sélection qui peut aboutir à la génération de variants devenus résistants au bactériophage. Dans le même temps, lors de la réplication en grand nombre du bactériophage, il se produit (naturellement et spontanément à faible fréquence) des erreurs de copie du matériel génétique qui conduisent à la génération d'une population hétérogène de phages. Cette hétérogénéité est, en fait, une réponse qui permet aux phages de s'adapter, par exemple, aux variations de séquence des récepteurs de leurs bactéries hôtes. Les bactéries et les bactériophages vivent donc dans un équilibre dynamique qui permet alternativement le développement d'une des deux espèces, tout en maintenant l'autre. Ces observations de laboratoire ont été validées sur le terrain comme notamment reporté par Faruque et al. lors d'une surveillance d'eaux de rivières indiennes régulièrement infestées par Vibrio cholerae [1]. Lorsque les eaux contiennent des bactéries $V$. cholerae on retrouve très peu de phages capables de lyser cellesci, par contre lorsque le nombre de bactéries est faible, coexistent plusieurs phages capables de lyser la souche initialement présente, et ainsi de suite.

\section{La phagothérapie}

Le contexte actuel de l'intérêt porté à nouveau à la phagothérapie est bien différent de celui qui avait vu sa naissance. Non seulement les connaissances sur les phages et leurs hôtes sont considérables, mais encore le nombre de modèles animaux disponibles et mieux maîtrisés est plus élevé. Cette revue se propose de parcourir la littérature la plus récente rapportant un traitement expérimental par les bactériophages organisé en fonction des bactéries pathogènes principalement rencontrée en milieu hospitalier, à savoir Escherichia coli, Staphylococcus aureus et Pseudomonas aeruginosa. Pour une revue plus complète le lecteur peut consulter les références [2-9] qui incluent aussi des articles reportant des essais de phagothérapie expérimentale sur d'autres espèces bactériennes.

\section{Traitement des infections à Escherichia coli}

Il n'est pas surprenant que les souches $E$. coli pathogènes soient les bactéries les plus couramment ciblées par des essais de phagothérapie expérimentale. D'une part les connaissances sur ces bactéries et sur leurs phages sont très étendues, et d'autre part il s'agit de pathogènes très répandus aussi bien chez l'homme que dans le monde animal. Parmis les souches d'E. coli, le sérotype $\mathrm{O} 157$ :H7 a fait l'objet de deux études récentes, l'une sur le tractus intestinal de souris et l'autre sur les ruminants [10,11]. Dans le cas de la souris, les auteurs ont choisi d'étudier l'efficacité d'un mélange (« cocktail ») de 3 phages après avoir démontré qu'en culture continue la souche pouvait rapidement résister à un phage (en 4 à $6 \mathrm{H})$ ou à 2 phages (en 10 à $30 \mathrm{H}$ ), et pas du tout à 3 phages (au moins jusqu'à $70 \mathrm{H}$ ). Deux jours après avoir introduit une dose de bactéries dans l'estomac des souris, certaines furent traitées par une simple dose du «cocktail » de phages et d'autres par une dose du même « cocktail » 1 fois par jour pendant 9 jours. Ce travail montre qu'une dose de phage administrée de manière journalière est beaucoup plus efficace qu'une simple dose. Cependant, bien que l'analyse des différentes parties de l'instestin montre qu'il n'y a plus de bactéries détectées dans la partie basse intestin grêle et dans le colon, il en reste dans la partie haute du petit intestin et dans le 
caecum, ainsi que dans les fèces. Dans le travail publié par Sheng et al., deux autres phages ont été utilisés tout d'abord chez la souris où une dose fût donnée oralement 1 fois par jour pendant 3 jours, une journée après l'infection orale par E. coli O157 :H7. Dans ce modèle, les auteurs montrent que les phages sont capables d'éliminer complètement la bactérie cible devenue indétectable dans les fèces 6 jours après le début du traitement. Par contre chez le jeune bœuf, dans un modèle de portage rectal, les mêmes bactériophages n'ont pas réussi à être aussi efficaces, permettant une diminution du nombre de bactéries introduites, mais pas leur élimination complète. Ces auteurs avancent dans leur discussion que l'effet d'une phagothérapie n'est pas le même selon que l'on utilise un modèle d'infection ou un modèle de portage chez l'animal. Dans une situation d'infection, les phages pourraient se révéler beaucoup plus efficaces en diminuant sur un temps court la quantité de bactéries infectieuses, permettant au système immunitaire de prendre le dessus sur le long terme. Alors que dans un modèle de portage, les phages seraient seuls à lutter contre des bactéries (qui sont accoutumées à leur hôte), ayant alors moins de chance d'éliminer celles-ci. On verra plus loin que les meilleurs résultats obtenus par les phages le sont dans des modèles infectieux, renforçant cette hypothèse (voir par exemple Xie et al., [12], pour le cas de diarrhées à $E$. coli). Dans l'éventualité où cette hypothèse se vérifiait, alors les phages ne seraient pas utiles dans l'éradication des bactéries infectieuses portées par les animaux, mais resteraient utilisables lors du traitement de produits dérivés (viande, lait,...) dans le but de diminuer le niveau de contamination de ces produits [13,14]. D'ailleurs une telle application industrielle a été approuvée par le Food and Drug Administration en 2006, afin de diminuer le niveau de contamination de différents produits alimentaires destinés à la consommation humaine en utilisant un « cocktail » de 6 phages dirigés contre Listeria.

\section{Traitement des infections à Staphylococcus aureus}

S. aureus est un pathogène majeur responsable notamment d'un grand nombre d'infections nosocomiales. Sa capacité à résister à de nombreux antibiotiques en fait une cible privilégiée pour des essais de phagothérapie. Deux articles récents démontrent que les phages peuvent protéger des souris contre une infection létale à $S$. aureus, par voie intraveineuse ou par voie intra-peritonéale $[15,16]$. Dans les deux cas l'injection précoce des bactériophages après l'infection est requise pour une efficacité maximale. De plus, Capparelli et al. ont montré qu'une infection chronique établie par voie intraveineuse peut être éliminée après une injection unique effectuée 10 jours après le début de l'infection. Enfin, dans un modèle d'abcès chez le lapin, à l'aide d'une souche naturellement pathogène pour celui-ci, Wills et al., ont pu démontrer un effet prophylactique [17].

S. aureus, ainsi que toutes les bactéries possède une couche de peptidoglycane qu'un phage doit être capable de lyser lorsqu'il termine son cycle infectieux afin de disséminer sa descendance. Pour ce faire, le phage possède dans son génome une séquence codant une enzyme hydrolytique (dénommée lysine) qui présente la particularité d'être spécifique de la bactérie infectée. Chez les bactéries à Gram positif le peptidoglycane est directement accessible à partir du milieu extérieur, et certains auteurs se sont penchés sur l'utilisation de la protéine purifiée comme agent anti-bactérien [18]. Les résultats sont spectaculaires.

L'efficacité de ces enzymes est excellente, et bien qu'elles soient spécifiques, certaines le sont moins permettant d'envisager l'utilisation de la même protéine pour lutter contre une majorité des souches d'une même espèce [19]. L'avantage majeur de l'utilisation d'une telle protéine est évident : il s'agit d'un produit totalement identifié et bien caractérisé dont la production peut être standardisée. Par contre, comme pour les antibiotiques, il est probable que les bactéries finissent par trouver la parade, après une modification de leur paroi qui pourrait les rendre moins infectieuses. Cependant, à ce jour aucune étude n'a montré à quelle fréquence une telle résistance pourrait émerger. Enfin les lysines ne sont d'aucune efficacité contre les bactéries à Gram négatif dont le peptidoglycane est séparé du milieu extérieur par une membrane externe. 
Enfin signalons un dernier article dans lequel un bactériophage fût ajouté à une solution de Ringer utilisée en milieu hospitalier pour le nettoyage des mains [20]. Les résultats montrent que le nombre de staphylocoques est diminué d'un facteur 100. De même, ce bactériophage fût ajouté à une crème et son efficacité testée sur une boite de Pétri, révélant qu'il est toujours actif. Ces essais, certes à très faible échelle, visent clairement à initier une démarche d'introduction des bactériophages en milieu hospitalier afin de diminuer l'incidence des infections nosocomiales. D'ailleurs dans ce but, depuis quelques mois, l'hôpital SacréCœur de Montréal au Canada a décidé de participer à une étude utilisant des bactériophages dirigés contre $S$. aureus.

\section{Traitement des infections à Pseudomonas aeruginosa}

$P$. aeruginosa est un pathogène opportuniste qui lui aussi au fil des ans est devenu de plus en plus résistant aux antibiotiques. Ce pathogène est bien connu de tous les patients atteints de mucoviscidose. Outre les poumons, cette bactérie peut souvent infecter la peau. Dans une telle situation le site infectieux est directement accessible à une application des bactériophages. Chez la souris, la mise au point d'un modèle d'infection cutanée suite à une blessure thermique a permis de démontrer que les bactériophages permettaient de sauver les animaux infectés par une dose létale de $P$. aeruginosa, à condition de les inoculer par voie intrapéritonéale [21]. En effet les auteurs ont comparé sur des animaux sains l'administration de phages par voies, sous-cutanée, intraveineuse et intrapéritonéale. Les bactériophages détectés au cours du temps dans le sang, le foie et la rate ont permis d'établir que l'injection intrapéritonéale était celle qui permettait aux batériophages de résider le plus longtemps dans le sang et les organes. Ainsi traités les animaux ont une chance de survie de $88 \%$. Un second article relate deux cas du traitement d'une infection à $P$. aeruginosa [22]. Un premier cas concerne le traitement d'une otite chronique chez un chien dont les multiples traitements antibiotiques ne venaient pas à bout, et le deuxième concerne une personne brûlée à $50 \%$ et dont les greffes ont dû être interrompues à cause de la survenue de l'infection. Dans ces deux cas il a été montré d'une part que les bactériophages utilisés se sont multipliés in situ au niveau du site infectieux et que d'autre part que l'infection a été éliminée, permettant de stopper tout traitement antibiotique pour l'animal et de reprendre avec succès les greffes de peau pour la personne. Enfin, bien que plus ancien, il est intéressant de citer un article qui semble être passé inaperçu dans les précédentes revues [23]. Il s'agit d'une étude menée en Égypte sur 30 patients brûlés à divers degrès, infectés par $P$. aeruginosa et ayant été traités sans succès par tous les antibiotiques disponibles. Un traitement par les bactériophages (pour lesquels aucune donnée n'est disponible) a été entrepris, et il en est conclu que dans $20 \%$ des cas une rémission totale a été observée, dans un peu plus de $40 \%$ des cas une nette amélioration a été constatée, alors que les $40 \%$ restant ne montraient qu'une faible ou pas d'amélioration. Les auteurs concluent que les bactériophages ne doivent pas être utilisés en routine pour tous les grands brûlés, mais réservés aux cas présentant une résistance aux antibiotiques. On ne peut souscrire à une telle conclusion.

\section{Synthèse :}

Les bactériophages lytiques sont les ennemis naturels des bactéries et probablement les plus efficaces. Ils représentent en quelque sorte une forme de lutte biologique. Est-ce que cette arme biologique pourra bénéficier à l'homme ? Cette question est l'objet du débat autour de leur utilisation en médecine. Afin d'alimenter ce débat au niveau scientifique, on peut aujourd'hui affirmer que les bactériophages qui sont capables de lyser des bactéries in vitro sur une boite de Pétri ne sont pas tous capables de faire de même in vivo poussant les chercheurs à identifier les candidats les plus virulents. Ici se pose un premier obstacle. Comment identifier les phages les plus virulents in vivo ? La plupart des auteurs se base sur le spectre d'activité des phages. Plus ce spectre est large plus les chances sont que ce phage soit 
efficace in vivo, sans que cela ait été clairement démontré. D'autres testent un nombre limité de phages et poursuivent leurs études avec le meilleur d'entre eux. Il serait certainement très utile de mettre au point une méthode permettant de discriminer rapidement les phages les moins virulents pour ne retenir que les meilleurs candidats. Malgré cela, les expériences se révèlent globalement très positives.

Faut-il craindre que les phages isolés de l'environnement possèdent des caractéristiques « indésirables » pour les personnes traitées ? La réponse sans pouvoir être absolument négative (il n'y a pas de risque zéro) est probablement non. D'une part seuls les phages lytiques devraient être utilisés, et d'autre part le coût du séquençage du génome d'un phage est aujourd'hui si faible que l'on pourra très prochainement séquencer rapidement le génome entier des phages potentiellement utilisables en thérapeutique. Ces données permettront d'écarter rapidement les phages pour lesquels on trouverait des homologies de séquences avec des gènes codant pour des protéines potentiellement «indésirables ».

Les différents articles cités ici font appel à différents modèles animaux. Ils ne sont pas encore suffisamment nombreux mais il est toutefois possible de distinguer deux situations. D'une part les modèles d'infections par injection intraveineuse des bactéries, nécessitant une application rapide des bactériophages, et les modèles d'infections et de colonisation par des voies plus ou moins naturelles (infections intestinales par exemple). Si d'une part les modèles d'infection par voie intraveineuse ont l'avantage de démontrer que les bactériophages sont efficaces in vivo, ils se révèlent assez limitant pour d'autres études. Par contre les modèles d'infection/colonisation présentent l'avantage de permettre des études sur des temps plus longs, mais ils ne se soldent pas toujours par l'éradication des bactéries. Il semble donc qu'il soit encore nécessaire de développer des modèles animaux, notamment d'infections chroniques, qui permettront de mieux appréhender l'efficacité des bactériophages dans des situations plus proches de celles pour lesquelles on envisage, dans un premier temps, des traitements chez l'homme. En effet, rien ne remplacera les antibiotiques tant que ceux-ci se révèleront efficaces, ce qui est encore le cas au quotidien dans la grande majorité des cas. Par contre, pour les situations de résistance et à la lumière des résultats expérimentaux, l'utilisation des bactériophages peut raisonnablement être envisagée.

\section{Conclusion :}

A l'aube du XXI ${ }^{\text {ème }}$ siècle, il apparaît ainsi nécessaire d'effectuer un transfert entre la phagothérapie couramment utilisée dans certains pays de l'Europe de l'Est depuis des décennies et les résultats encourageants obtenus dans les laboratoires de recherche notamment dans les pays de l'Europe de l'Ouest. Ceci nécessitera que des décisions réglementaires soient prises afin de délimiter au mieux des essais thérapeutiques chez l'homme, dans des conditions convenables et non pas uniquement dans des situations compassionnelles qui sont toujours sujettes à caution. Bien sûr il ne faut pas aller ni trop vite ni trop loin, mais il faut progresser. Il y a aujourd'hui une dynamique aussi bien du côté médical que du côté recherche, et il serait regrettable de ne pas saisir cette opportunité pour quitter la situation d'immobilisme qui a prévalu depuis plus de 20 ans. Signalons ici le travail exemplaire qui vient d'être entrepris en Belgique afin de mettre sur pied des essais cliniques en respectant toutes les réglementations en vigueur (ce qui ne fût pas sans peine !) [24].

Plusieurs infections pour lesquelles le diagnostic n'est pas vital et qui sont aujourd'hui connue pour aboutir la plupart du temps à une impasse antibiotique, pourraient être le terrain d'essais thérapeutiques contrôlés, pour qu'ainsi on rompe le cercle qui empêche le corps médical de pratiquer de manière autorisée et éclairée une approche thérapeutique reconnue comme efficace dans d'autres pays. Les maladies n'ont jamais connues de frontières, pourquoi en serait-il le cas pour les thérapies? 


\section{Références Bibliographiques}

1 Faruque SM, Naser IB, Islam MJ, et al. Seasonal epidemics of cholera inversely correlate with the prevalence of environmental cholera phages. Proceedings of the National Academy of Sciences of the United States of America 2005; 102: 1702-7.

2 Biswas B, Adhya S, Washart P, et al. Bacteriophage therapy rescues mice bacteremic from a clinical isolate of vancomycin-resistant Enterococcus faecium. Infect Immun 2002; 70: 20410.

3 Borysowski J, Weber-Dabrowska B, Gorski A. Bacteriophage endolysins as a novel class of antibacterial agents. Experimental biology and medicine (Maywood, NJ 2006; 231: 366-77.

4 Carlton RM, Noordman WH, Biswas B, de Meester ED, Loessner MJ. Bacteriophage P100 for control of Listeria monocytogenes in foods: genome sequence, bioinformatic analyses, oral toxicity study, and application. Regul Toxicol Pharmacol 2005; 43: 301-12.

5 Entenza JM, Loeffler JM, Grandgirard D, Fischetti VA, Moreillon P. Therapeutic effects of bacteriophage Cpl-1 lysin against Streptococcus pneumoniae endocarditis in rats.

Antimicrobial agents and chemotherapy 2005; 49: 4789-92.

6 Kutter ESA. Bacteriophages, Biology and Applications. Boca Raton, FL, USA: CRC Press Inc, 2005.

7 Merril CR, Scholl D, Adhya SL. The prospect for bacteriophage therapy in Western medicine. Nat Rev Drug Discov 2003; 2: 489-97.

8 Nakai T, Park SC. Bacteriophage therapy of infectious diseases in aquaculture. Res Microbiol 2002; 153: 13-8.

9 Summers WC. Bacteriophage therapy. Annual review of microbiology 2001; 55: 437-51.

10 Sheng H, Knecht HJ, Kudva IT, Hovde CJ. Application of bacteriophages to control intestinal Escherichia coli O157:H7 levels in ruminants. Applied and environmental microbiology 2006; 72: 5359-66.

11 Tanji Y, Shimada T, Fukudomi H, Miyanaga K, Nakai Y, Unno H. Therapeutic use of phage cocktail for controlling Escherichia coli O157:H7 in gastrointestinal tract of mice. Journal of bioscience and bioengineering 2005; 100: 280-7.

12 Xie H, Zhuang X, Kong J, Ma G, Zhang H. Bacteriophage Esc-A is an efficient therapy for Escherichia coli 3-1 caused diarrhea in chickens. The Journal of general and applied microbiology 2005; 51: 159-63.

13 O'Flynn G, Ross RP, Fitzgerald GF, Coffey A. Evaluation of a cocktail of three bacteriophages for biocontrol of Escherichia coli O157:H7. Applied and environmental microbiology 2004; 70: 3417-24.

14 Rees CE, Dodd CE. Phage for rapid detection and control of bacterial pathogens in food. Advances in applied microbiology 2006; 59: 159-86.

15 Capparelli R, Parlato M, Borriello G, Salvatore P, Iannelli D. Experimental phage therapy against Staphylococcus aureus in mice. Antimicrobial agents and chemotherapy 2007; 51: 2765-73.

16 Matsuzaki S, Yasuda M, Nishikawa H, et al. Experimental protection of mice against lethal Staphylococcus aureus infection by novel bacteriophage phi MR11. J Infect Dis 2003; 187: 613-24.

17 Wills QF, Kerrigan C, Soothill JS. Experimental bacteriophage protection against Staphylococcus aureus abscesses in a rabbit model. Antimicrobial agents and chemotherapy 2005; 49: 1220-1.

18 Fischetti VA. Bacteriophage lytic enzymes: novel anti-infectives. Trends Microbiol 2005; 13: 491-6.

19 O'Flaherty S, Coffey A, Meaney W, Fitzgerald GF, Ross RP. The recombinant phage lysin LysK has a broad spectrum of lytic activity against clinically relevant staphylococci, including methicillin-resistant Staphylococcus aureus. Journal of bacteriology 2005; 187 : 7161-4. 
20 O'Flaherty S, Ross RP, Meaney W, Fitzgerald GF, Elbreki MF, Coffey A. Potential of the polyvalent anti-Staphylococcus bacteriophage $\mathrm{K}$ for control of antibiotic-resistant staphylococci from hospitals. Applied and environmental microbiology 2005; 71: 1836-42. 21 McVay CS, Velasquez M, Fralick JA. Phage therapy of Pseudomonas aeruginosa infection in a mouse burn wound model. Antimicrobial agents and chemotherapy 2007; 51: 1934-8. 22 Marza JA, Soothill JS, Boydell P, Collyns TA. Multiplication of therapeutically administered bacteriophages in Pseudomonas aeruginosa infected patients. Burns 2006; 32: 644-6.

23 Abul-Hassan HS, K. E-T, Massoud B, R. G. Bacteriophage therapy of Pseudomonas burn wound sepsis. Annals of the MBC 1990; 3: 262.

24 Verbeken G, De Vos D, Vaneechoutte M, Merabishvili M, Zizi M, Pirnay JP. European regulatory conundrum of phage therapy. Future microbiology 2007; 2: 485-91. 